\title{
Tracing the phase of focused broadband laser pulses
}

\author{
Dominik Hoff ${ }^{1, *}$, Michael Krüger $^{2,4}$, Lothar Maisenbacher ${ }^{3}$, A. Max Sayler ${ }^{1}$, Peter \\ Hommelhoff $^{4}$ and Gerhard G. Paulus ${ }^{1}$ \\ ${ }^{1}$ Helmholtz-Institut Jena and Institut für Optik und Quantenelektronik, Friedrich-Schiller-Universität \\ Jena, Max-Wien-Platz 1, 07743 Jena, Germany \\ ${ }^{2}$ Department of Physics of Complex Systems, Weizmann Institute of Science, 234 Herzl St., Rehovot \\ 76100, Israel \\ ${ }^{3}$ Max-Planck-Institut für Quantenoptik, Hans-Kopfermann-Str. 1, 85748 Garching, Germany \\ ${ }^{4}$ Department Physik, Friedrich-Alexander-Universität Erlangen-Nürnberg (FAU), Staudtstr. 1, 91058 \\ Erlangen, Germany
}

\begin{abstract}
We present a three-dimensional measurement of the local focal phase in a focused broadband Gaussian laser beam and find strong deviations from the commonly assumed Gouy phase, with wide ramifications for ultrafast physics.
\end{abstract}

\section{Introduction}

Precise knowledge of the behaviour of the phase of light in the focus of a laser beam is fundamental to understand and control laser-driven processes. More than a hundred years ago the axial phase evolution for focused monochromatic light beams was described and is now commonly known as the Gouy phase [1]. Recent theoretical work has brought into question the validity of applying this monochromatic phase formulation to the broadband laser pulses becoming ubiquitous today [2]. The need for investigation of the spatial behaviour of the local carrier-envelope phase (CEP) is also underscored by experimental studies, which suggest deviations from the monochromatic Gouy phase (see for instance $[3])$.

\section{Method}

Based on electron back-scattering at sharp nanometre-scale metal tips, a method is available to measure light fields with sub-wavelength spatial resolution. We combine this effect with a high-precision pulse characterization to determine the random CEP of each laser shot, namely a phase meter based on above-threshold ionization [5], in this way achieving also sub-optical cycle time resolution [4].

We report a quantitative, direct, three-dimensional measurement of the focal CEP evolution of a few-cycle (4-fs), near-infrared laser beam, spanning a range of seven times

\footnotetext{
* Corresponding author: dominik.hoff@uni-jena.de
} 
the Rayleigh range along the propagation axis and one and a half times the local beam radius perpendicular to the optical axis [6].

The nanotip is scanned through the focus and photoelectron spectra are recorded at a set of points in space using a time-of-flight spectrometer, Fig. 1. The detected electrons are associated with the CEP of the respective laser shot producing them. The CEP modulation of the tagged spectra reveals the local phase from which the spatial evolution can be determined.

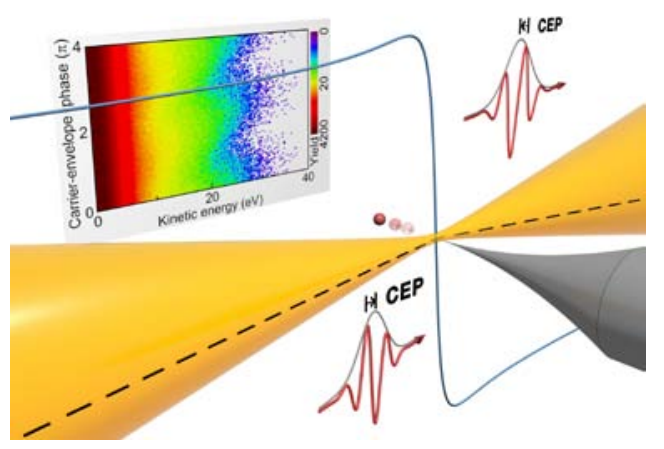

Fig. 1. Scheme of the measurement: the nanotip is scanned along the focus where at each position CEP-tagged spectra of the emitted electrons are recorded (background).

\section{Results}

The results are summarized in Fig. 2, which shows the CEP evolution on the optical axis (left), obtained with two different nanotips. The measurement clearly deviates from the arctan-like Gouy phase (dashed). The right panel depicts the off-axis CEP behaviour. We find that in addition to the deviations from the Gouy phase on axis, a transverse dependence can also be observed. Both can be reproduced well with the model described in Ref. [2], where the deviations can be attributed to the spectral geometry of the input beam. This can be used to independently estimate the CEP-profile-determining parameter via the spectrally resolved beam profile. The variability of the spectral beam geometry dependent focal CEP, see Fig. 3, could also be used to design the interaction reaction such that, for instance, the high harmonic yield is maximized [7].
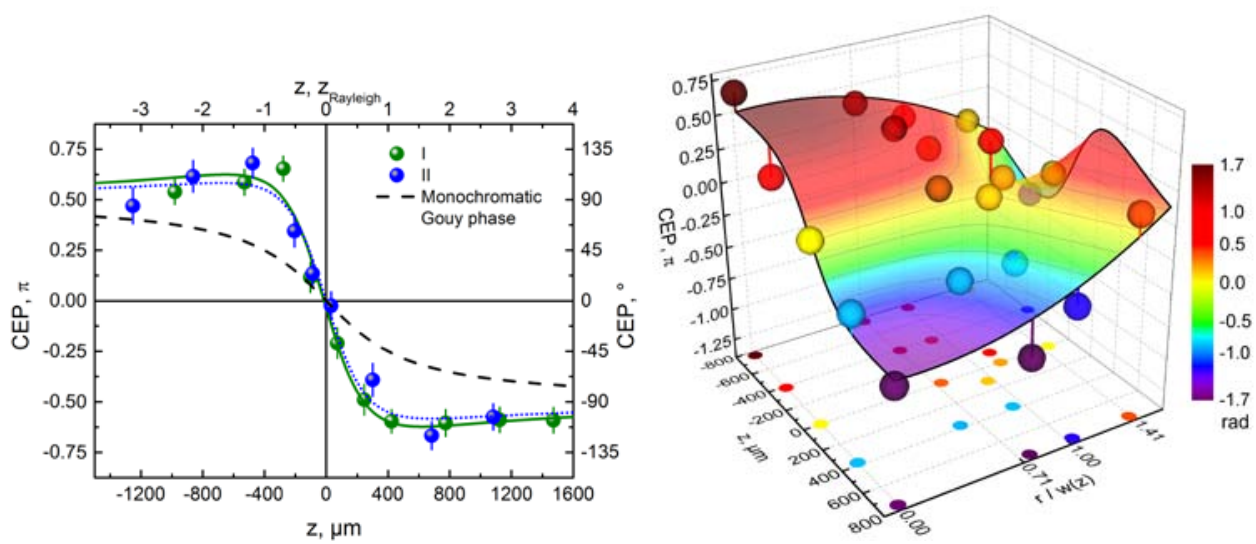

Fig. 2. Measured carrier-envelope phase (CEP) evolution of focused, 4-fs, laser pulses on the optical axis (left) and off the optical axis (right) fit by a theoretical mode 


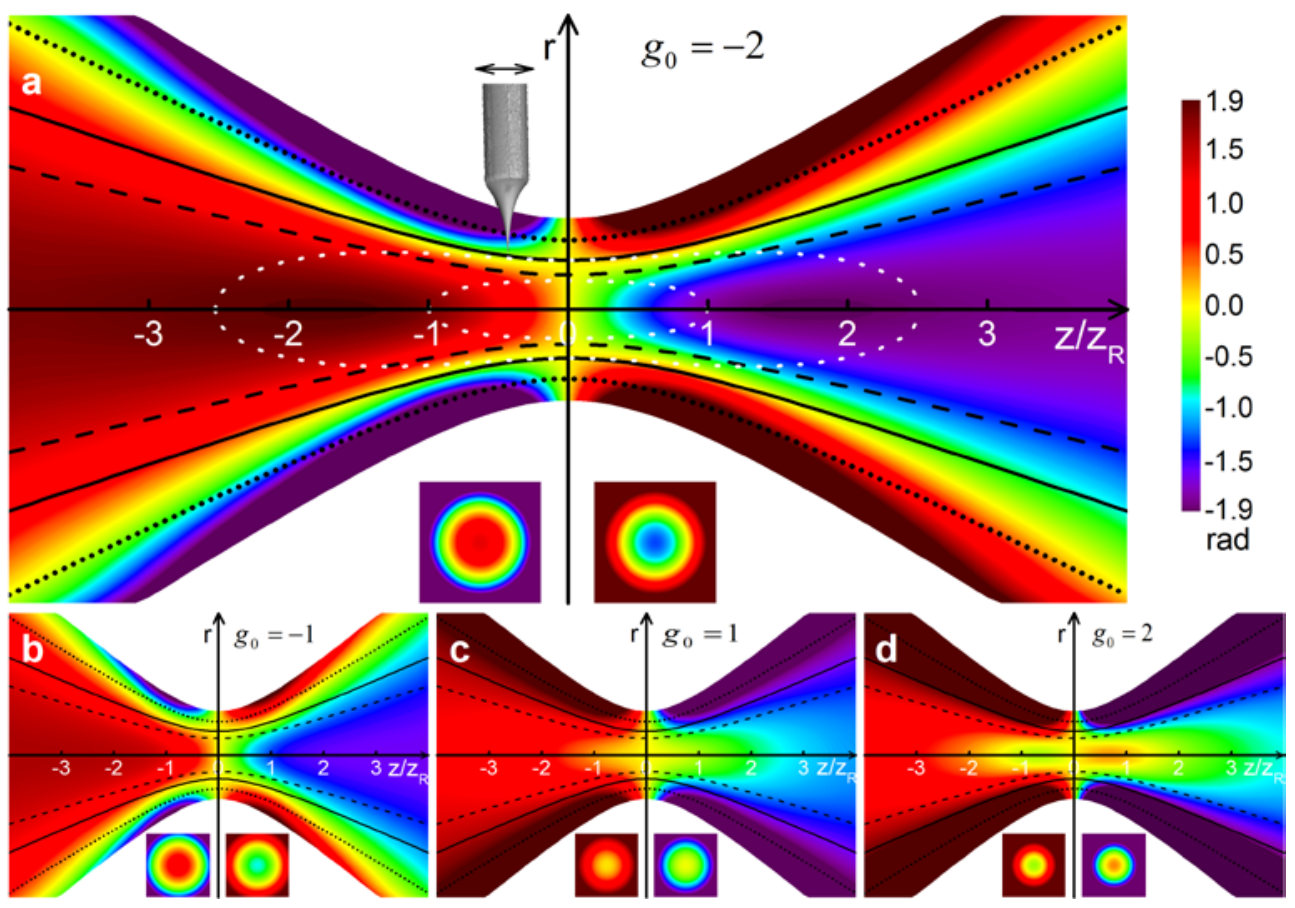

Fig. 3. Calculated focal CEP evolution for different spectral input beam geometries (a-d), described by parameter $\mathrm{g}_{0}$, the insets show beam cross sections at $\pm 0.5 \mathrm{z}_{\mathrm{R}}$, see references for details $[2,6,7,8]$

\section{Conclusion}

Our observations show that significant deviations from the commonly assumed Gouy phase behaviour need to be taken into account for focused, broadband laser pulses. This finding can be crucial for the measurement and understanding of many attosecond processes and accounting for these effects may be required for proper interpretation. Moreover, manipulating these effects has the potential to enhance the desired dynamics. Therefore, we expect wide ramifications for all broadband laser-matter interactions, which are used in fields like high-harmonic and attosecond pulse generation, femtochemistry, optical coherence tomography and light-wave electronics.

\section{References}

1. L. G. Gouy, C. R. Acad. Sci. Paris 110, 1251-1253 (1890)

2. M. A. Porras, Opt. Lett. 34, 1546-1548 (2009)

3. T. Tritschler, K. Hof, M. Klein, \& M. Wegener, Opt. Lett. 30, 753-755 (2005)

4. M. Krüger, M. Schenk \& P. Hommelhoff, Nature 475, 78-81 (2011)

5. N. G. Johnson, et al., Phys. Rev. A 83, 013412 (2011)

6. D. Hoff, M. Krüger, L. Maisenbacher, A.M. Sayler, G.G. Paulus, P. Hommelhoff, Nat. Phys. 13947 - 51 (2017)

7. D. Hoff, M. Krüger, L. Maisenbacher, A.M. Sayler, P. Hommelhoff, G.G. Paulus, J. Opt. 19124007 (2017)

8. D. Hoff, M. Krüger et al., http://arxiv.org/abs/1805.11531 